\title{
High proportions of asymptomatic and submicroscopic Plasmodium vivax infections in a peri-urban area of low transmission in the Brazilian Amazon
}

\author{
Anne C. G. Almeida ${ }^{1,2^{* \dagger}}$, Andrea Kuehn ${ }^{3 \dagger}$, Arthur J. M. Castro ${ }^{2,4}$, Sheila Vitor-Silva', Erick F. G. Figueiredo ${ }^{1}$, \\ Larissa W. Brasil ${ }^{1}$, Marcelo A. M. Brito ${ }^{1,2}$, Vanderson S. Sampaio ${ }^{5}$, Quique Bassat ${ }^{3,6,7}$, Ingrid Felger ${ }^{8,9}$, \\ Wanderli P. Tadei ${ }^{4}$, Wuelton M. Monteiro ${ }^{1,2^{*}}$, Ivo Mueller ${ }^{3,10}$ and Marcus V. G. Lacerda ${ }^{1,2,11}$
}

\begin{abstract}
Background: Population-based studies conducted in Latin America have shown a high proportion of asymptomatic and submicroscopic malarial infections. Considering efforts aiming at regional elimination, it is important to investigate the role of this asymptomatic reservoir in malaria transmission in peri-urban areas. This study aimed to estimate the prevalence of Plasmodium spp. and gametocyte burden on symptomatic and asymptomatic infections in the Brazilian Amazon.

Results: Two cross-sectional household surveys (CS) were conducted including all inhabitants in a peri-urban area of Manaus, western Amazonas State, Brazil. Malaria parasites were detected by light microscopy (LM) and qPCR. Sexual stages of Plasmodium spp. were detected by LM and RT-qPCR. A total of 4083 participants were enrolled during the two surveys. In CS1, the prevalence of Plasmodium vivax infections was 4.3\% (86/2010) by qPCR and 1. $6 \%(32 / 2010)$ by LM. Fifty percent (43/86) of $P$. vivax infected individuals (qPCR) carried $P$. vivax gametocytes. In CS2, $3.4 \%$ (70/2073) of participants had qPCR-detectable P. vivax infections, of which 42.9\% (30/70) of infections were gametocyte positive. The $P$. vivax parasite density was associated with gametocyte carriage $(P<0.001)$. Sixty-seven percent of $P$. vivax infected individuals and $53.4 \%$ of $P$. vivax gametocyte carriers were asymptomatic.

Conclusions: This study confirms a substantial proportion of asymptomatic and submicroscopic $P$. vivax infections in the study area. Most asymptomatic individuals carried gametocytes and presented low asexual parasitemia. This reservoir actively contributes to malaria transmission in the Brazilian Amazon, underscoring a need to implement more efficient control and elimination strategies.
\end{abstract}

Keywords: Malaria, Plasmodium vivax, Asymptomatic infection, Submicroscopic infection, Gametocyte carriage

\section{Background}

The past two decades have witnessed impressive success in the reduction of malaria incidence in Latin America, with the number of confirmed cases decreasing by $31.0 \%$ from 2000 to 2015. Nevertheless, malaria remains a major public health concern in endemic countries, with

\footnotetext{
* Correspondence: annealmeida.gb@gmail.com; wueltonmm@gmail.com ${ }^{\dagger}$ Equal contributors

${ }^{1}$ Fundação de Medicina Tropical Dr. Heitor Vieira Dourado (FMT-HVD), Av. Pedro Teixeira, N.25, Dom Pedro, Manaus, Amazonas CEP: 69040-000, Brazil

Full list of author information is available at the end of the article
}

approximately 90 million people being at risk of infection and an estimated 216 million cases worldwide in 2016 [1]. In 2013 more than 40\% of malaria cases in Latin America occurred in Brazil and, within that country, more than $99 \%$ of all cases were concentrated in the Amazon region [2]. From 2005 to 2015, malaria incidence in Brazil decreased considerably, as evidenced by reductions in Annual Parasite Index (79.2\%) and total number of cases $(76.5 \%)$. This trend was accompanied by an even more pronounced decline in the proportion of Plasmodium falciparum, with Plasmodium vivax 
predominance progressively increasing to peak at $88.4 \%$ of total malaria cases in 2015 [3]. Such an impressive reduction of cases is likely attributable to the governmental program focusing on early case detection and treatment [4]. Despite the relative success of control efforts, malaria transmission still occurs in the Amazon region with major outbreaks recorded in 1999 and 2005 that may have been in part due to deforestation, mining activities and new unplanned rural settlements resulting in environmental changes favouring malaria transmission $[2,5]$. After the 1970s, a large migratory influx from the rural zones to the peripheries of bigger cities in the Amazon basin resulted in a precarious and uncontrolled urbanization process, increasing human exposure to anopheline vectors in periurban areas [6, 7]. In Brazilian Amazon, Anopheles darlingi is the main malaria vector $[8,9]$.

Latin America is considered a low to moderate malaria transmission region. Recent population based surveys reported prevalence rates below $6.0 \%$ by LM [10-14]. PCR analyses revealed higher prevalences, ranging between $5.0-17.0 \%$ [10-14]. In some studies the majority of infected individuals did not show malariarelated symptoms $[12,15]$. These results suggest that a large proportion of infections was submicroscopic and asymptomatic and would not be detected by routine diagnostic methods, although asymptomatic infections are believed to substantially contribute to malaria transmission. Thus, in this renewed elimination era, it may seem appropriate to reconsider the role of the asymptomatic reservoir as a likely source of transmission, especially in low transmission settings. Specifically and actively targeting this reservoir, rather than relying on passive detection of clinically symptomatic malaria infections, may be essential to achieve local elimination.

Most studies examining prevalence of Plasmodium spp. in the Amazon region were carried out in small rural or riverine settlements. Additionally, very few studies assessed the prevalence and density of gametocytes, the parasite stage responsible for transmission from human to mosquito vectors. This study aimed to determine the prevalence of Plasmodium spp. and the gametocyte burden in symptomatic and asymptomatic infections in a peri-urban area in the Brazilian Amazon.

\section{Methods}

In order to estimate the reservoir of Plasmodium spp. infected individuals contributing to malaria transmission, we conducted two large-scale cross-sectional surveys in a peri-urban area of Manaus, located in the western part of the Amazonas State, Brazil. The presence of sexual parasite stages in both symptomatic and asymptomatic infected individuals was also investigated.

\section{Study site}

The municipality of Manaus is located on the river Rio Negro banks, in the northeast of the Amazonas State. The municipality has an estimated 2,094,301 inhabitants of whom the great majority resides in urban and peri-urban areas [16]. Intensive migration, as well as persisting challenges to maintaining an adequate epidemiological and entomological surveillance, are contributing to the actively ongoing sustained malaria transmission in the region. Risk of infection varies between low and high risk in rural and peri-urban areas. Highest incidence of malaria cases is commonly observed from May to September, in the dry season when water levels are declining [17].

The three communities in which this study was conducted, Brasileirinho, Ipiranga and Puraquequara, are located in the peri-urban area of Manaus (Fig. 1). The main occupation is in the agricultural sector including pisciculture. Many people travel to the city daily for work. There are many places for religious conventions and leisure activities, where people from the city stay for the weekend and public holidays. Malaria infections among these visitors from non-endemic areas of Manaus are common. Sanitation and garbage collection services are not available, water is collected from natural springs or rivers as well as received from a city service. According to a census performed by a field team of Fundação de Medicina Tropical Dr. Heitor Vieira Dourado (FMT-HVD) the population of the study area (Brasileirinho, Ipiranga and the northern part of Puraquequara, adjacent to Brasileirinho) was estimated at around 2400 inhabitants in 2012. Each community has access to one malaria clinic specifically conducting malaria diagnosis by microscopy and treatment of cases. Health agents based in this clinic regularly visit homes in order to ensure early detection and treatment of cases. Sporadically they perform active detection of febrile cases in the neighbourhood of confirmed cases.

\section{Study population and survey method}

Two cross-sectional surveys were conducted on a household-basis, including all inhabitants of all ages of the study area that were willing to participate in the study. In case of absence of any family members, the field team returned to the respective house for a second time. For each study participant a questionnaire was completed, containing personal information (age, gender, occupation, pregnancy, history of travel), information on malaria preventive measures, previous malaria episodes and current health status. The first cross-sectional survey (CS1) was conducted from mid-November 2012 to beginning of January 2013, at 


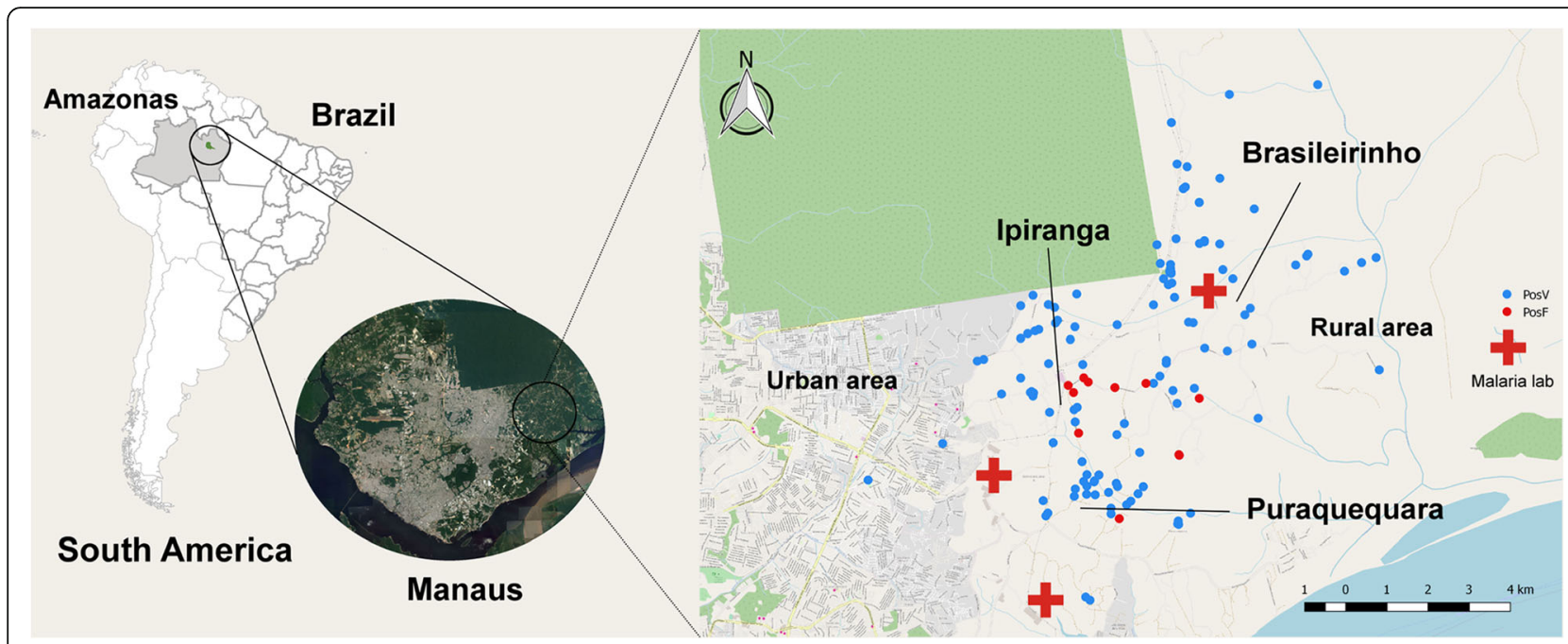

Fig. 1 Study area and distribution of malaria infected individuals (red: P. falciparum, blue: P. vivax) enrolled at cross-sectional surveys, detected by qPCR

the beginning of the rainy season, and the second one (CS2) from the beginning of August 2013 to mid-September 2013, at the end of the dry season.

\section{Sample collection}

Upon participant enrolment, a $300 \mu$ blood sample was collected from each patient via finger puncture using Microtainer tubes containing EDTA and sodium fluoride (Becton Dickinson, NJ, USA). In infants, blood was obtained by puncture of the heel or toe. Within $1 \mathrm{~h}$ of collection, $50 \mu \mathrm{l}$ of blood was transferred into a reaction tube containing $250 \mu \mathrm{l}$ of RNAprotect ${ }^{\circ}$ (QIAGEN, Hilden, Germany) in order to preserve RNA for downstream analyses [18] and $200 \mu \mathrm{l}$ of whole blood were transferred to another reaction tube. Samples were maintained in cooling boxes until arrival in the laboratory, where the $200 \mu \mathrm{l}$ sample was separated into red blood cell (RBC) pellet and plasma. All samples were frozen at $-80{ }^{\circ} \mathrm{C}$ until further processing. If the collected blood volume was $<250 \mu \mathrm{l}$, the actual volume was recorded.

In order to assess the submicroscopic proportion of infections, during CS1 two thick blood smears were prepared for all participants. One slide was analysed at the local malaria outpost (for diagnostics purposes only) and the second was analysed by a microscopist at FMT-HVD for confirmation of species and parasitemia. In cases of a positive result, participants were treated by health post staff within $24 \mathrm{~h}$ according to the national guidelines of the Ministry of Health [19]. During CS2 only in the case of symptoms related to malaria a thick blood smear was prepared for diagnosis according to the national guidelines. Participants with positive result received prompt treatment.

\section{Classification of infection status}

All participants were tested for $P$. vivax and $P$. falciparum by qPCR (and in CS1 additionally by LM) to determine positivity of infection. Individuals with body temperature above $37.5{ }^{\circ} \mathrm{C}$ at blood collection or in the past $48 \mathrm{~h}$ were considered as febrile. An asymptomatic infection was defined as the presence of a malarial infection in the absence of fever and any other malaria related symptoms (chills, sweating, headache, vomit, abdominal pain) at the moment of sample collection, or anytime during the preceding $48 \mathrm{~h}$. Individuals developing symptoms within 30 days after blood collection (data obtained from SIVEP-Malaria database, Information System for Epidemiological Surveillance of Malaria of the Brazilian Ministry of Health) were also considered symptomatic and therefore excluded from the asymptomatic group.

\section{Detection of Plasmodium spp. parasites by light microscopy}

Thick blood smears were Giemsa-stained according to World Health Organization guidelines [20]. Numbers of asexual blood stage parasites and gametocytes were determined per 200 leukocytes and parasites per $\mu \mathrm{l}$ were calculated using an assumed density of 6000 leukocytes per $\mu \mathrm{l}$ [21]. For quality control purposes, all slides with positive results and $10 \%$ of all negative slides were read by a second microscopist. In case of a positive/negative discrepancy, or when parasitemias were in disagreement by more than $25.0 \%$, the slides were reread by a third microscopist and only the two most similar results were used for calculation of final parasite density. All PCR positive samples were examined at least twice by LM. 


\section{DNA and RNA extraction}

Pelleted RBCs obtained from $200 \mu$ l blood were resuspended in PBS and genomic DNA was extracted using FavorPrep $^{\text {TM }}$ 96-well Genomic DNA Kit (Favorgen, Ping-Tung, Taiwan) according to the manufacturer's instructions. DNA was eluted with $2 \times 100 \mu$ lof elution buffer and stored at $-20{ }^{\circ} \mathrm{C}$ until analysed by PCR. If the amount of whole blood available for DNA extraction was $\leq 100 \mu \mathrm{l}$, the DNA volume was vacuum concentrated (Concentrator 5301, Eppendorf, Hamburg, Germany) according to the volume originally collected. RNA from whole blood stored in RNAprotect was extracted by means of the RNeasy ${ }^{\circ}$ Plus 96 kit (Qiagen, Hilden, Germany) as described elsewhere [18].

\section{Detection of Plasmodium spp. by qPCR and detection of gametocyte-specific transcripts by RT-qPCR}

All DNA samples were subjected to QMAL Taqman qPCR to detect Plasmodium spp. by targeting a conserved region of the $18 \mathrm{~S}$ rRNA gene [18]. QMALpositive samples were further analysed by Taqman qPCR assays detecting species-specific sequences of $18 \mathrm{~S}$ rRNA gene of $P$. falciparum and $P$. vivax, as previously described $[18,22]$. For detection of $P$. falciparum a modified reverse primer was used (Additional file 1: Table S1) [23]. For quantification of $18 S$ rRNA gene copy numbers, in each experiment three dilutions of plasmids containing the respective region targeted were included in triplicates $\left(10^{2}, 10^{4}\right.$ and $10^{6}$ copies/ $\left.\mu \mathrm{l}\right)$. All samples positive for $P$. vivax and/or $P$. falciparum were included in RT-qPCR assays detecting the respective transcripts of $p 25$ gene (pvs 25 in P. vivax and $p f s 25$ in $P$. falciparum), which is specifically expressed in gametocytes. For quantification of $p v s 25$ and $p f s 25$ transcripts, standard plasmids containing the region targeted by the RT-qPCR were included in each run. All qPCR and RTqPCR assays were ran in the 7500 Fast Real-Time PCR System (Applied Biosystems, California, USA). Primer and probe sequences, composition of reaction mixes, PCR profiles and the detection limit for each assay are listed in Additional file 1: Table S1, Additional file 2: Table S2 and Additional file 3: Table S3.

\section{Statistical analyses}

Questionnaire data were imported into databases using Cardiff TeleForm version 10.4.1 (Cardiff Software). Individual databases were combined in Microsoft Access 2010. Statistical analyses were performed with STATA ${ }^{\circ}$ 13 (StataCorp LP, College Station, TX, USA). Differences in participant characteristics, parasite prevalence and carriage of gametocyte between the two surveys were analysed by Chi-square $\left(\chi^{2}\right)$ test (replaced by Fisher's exact test if necessary). Univariable logistic regression was used to determine risk factors for $P$. vivax infection and gametocyte positivity. The crude odds ratios (OR) with their respective $95 \%$ confidence intervals $(95 \% \mathrm{CI})$ were determined considering $P$. vivax infection and gametocyte carriage as the dependent variables. Logistic regression was used for the multivariable analyses and the adjusted ORs (AOR) with 95\% CI were also calculated. All variables associated with the outcomes at a significance level of $P<0.20$ in the univariable analysis were included in the multivariable analysis. Median comparisons for copy number between surveys and between symptomatic and asymptomatic individuals were made using Mann-Whitney test. Differences were considered statistically significant for $P<0.05$. Association between $P$. vivax parasite density and $P$. vivax gametocyte density was analysed by linear regression.

\section{Results}

\section{Study population}

A total of 4083 participants were included in the two surveys: 1047 people were surveyed only once during the beginning of the rainy season in 2012 (CS1), 1110 only during the 2013 dry season (CS2) and 963 at both time points (Table 1). Overall, 2138 participants were male (52.4\%), 507 were aged under 5 years (12.4\%), 710 were $5-11$ years-old (17.4\%), 502 were $12-17$ years-old (12.3\%), 1974 were $18-59$ years-old $(48.3 \%)$ and 388 were 60 years-old or older (9.5\%), with no significant differences regarding age $\left(\chi^{2}=2.815, d f=4, P=0.589\right)$ or gender $\left(\chi^{2}=0.119, d f=1, P=0.731\right)$ between surveys. Besides pre-school (15.0\%) and school children (24.4\%), $15.4 \%$ of participants were adults working in agriculture/ fishing, $14.3 \%$ worked in an office or pursued high education studies, while $15.6 \%$ were house wives and $5.0 \%$ retired (Table 1). Ten percent were engaged in other professions or unemployed. The majority of participants had lived in the village for longer than two months (CS1: 93.8\%, CS2 97.8\%, $\chi^{2}=40.359, d f=1, P<0.0001$ ) and few had travelled outside the village in the month prior to the survey (CS1: 9.4\%, CS2: 9.0\%, $\chi^{2}=0.196$, $d f=1, P=0.658$ ) (Table 1).

The majority of participants lived in households that had benefited from vector control activities. In CS1 73.7\% (1482/2010) of participants were using LLINs (long-lasting insecticide-treated nets) and/or lived in a house that was treated with indoor spraying of residual insecticides (IRS) in the preceding six months, in CS2 it was $54.5 \%(1131 / 2073)$ of participants $\left(\chi^{2}=162.812\right.$, $d f$ $=1, P<0.0001)$. In CS1, 50.1\% (1008/2010) used LLINs, $56.7 \%(1140 / 2010)$ lived in an IRS treated house, and $33.1 \%(666 / 2010)$ of participants had benefited from both interventions. As LLIN distributions and IRS spraying campaigns were conducted in the months prior to CS1, the proportion of people that reported benefitting from either intervention was significantly higher in CS1 
Table 1 Characteristics of study participants

\begin{tabular}{|c|c|c|c|c|c|c|}
\hline \multirow[t]{2}{*}{ Variable } & \multicolumn{2}{|l|}{ CS1 } & \multicolumn{2}{|l|}{$\mathrm{CS} 2$} & \multirow[t]{2}{*}{$x^{2}$} & \multirow[t]{2}{*}{$P$-value } \\
\hline & $n$ & $\%$ & $n$ & $\%$ & & \\
\hline Total & 2010 & 100.0 & 2073 & 100.0 & & \\
\hline \multicolumn{7}{|l|}{ Demographic } \\
\hline \multicolumn{7}{|l|}{ Community } \\
\hline Ipiranga & 439 & 21.8 & 487 & 23.5 & \multirow[t]{3}{*}{34.507} & \multirow[t]{3}{*}{$<0.0001$} \\
\hline Brasileirinho & 1185 & 59.0 & 1049 & 50.6 & & \\
\hline Puraquequara & 386 & 19.2 & 537 & 25.9 & & \\
\hline \multicolumn{7}{|l|}{ Gender } \\
\hline Male & 1058 & 52.6 & 1080 & 52.1 & \multirow[t]{2}{*}{0.119} & \multirow[t]{2}{*}{0.731} \\
\hline Female & 952 & 47.4 & 993 & 47.9 & & \\
\hline \multicolumn{7}{|l|}{ Age group } \\
\hline$<5$ yrs & 256 & 12.7 & 251 & 12.1 & \multirow[t]{5}{*}{2.815} & \multirow[t]{5}{*}{0.589} \\
\hline$\geq 5-11$ yrs & 361 & 18.0 & 349 & 16.8 & & \\
\hline $12-17$ yrs & 253 & 12.6 & 249 & 12.0 & & \\
\hline $18-59$ yrs & 957 & 47.7 & 1017 & 49.1 & & \\
\hline$\geq 60 \mathrm{yrs}$ & 181 & 9.0 & 207 & 10.0 & & \\
\hline \multicolumn{7}{|l|}{ Occupation } \\
\hline Office worker and higher education student & 318 & 15.8 & 296 & 14.3 & \multirow[t]{7}{*}{45.221} & \multirow[t]{7}{*}{$<0.0001$} \\
\hline Agriculture/fishing & 349 & 17.4 & 277 & 13.4 & & \\
\hline Housewife & 289 & 14.4 & 346 & 16.7 & & \\
\hline Retired & 106 & 5.3 & 97 & 4.7 & & \\
\hline School children & 481 & 23.9 & 484 & 23.4 & & \\
\hline Infants and preschool children & 312 & 15.5 & 300 & 14.5 & & \\
\hline Unemployed/ other & 154 & 7.7 & 269 & 13.0 & & \\
\hline \multicolumn{7}{|l|}{ More than 2 months in the area } \\
\hline Yes & 1866 & 93.8 & 2017 & 97.8 & \multirow[t]{2}{*}{40.359} & \multirow[t]{2}{*}{$<0.0001$} \\
\hline No & 124 & 6.2 & 46 & 2.2 & & \\
\hline \multicolumn{7}{|l|}{ Recent travel (last month) } \\
\hline Yes & 188 & 9.4 & 186 & 9.0 & \multirow[t]{2}{*}{0.196} & \multirow[t]{2}{*}{0.658} \\
\hline No & 1814 & 90.6 & 1883 & 91.0 & & \\
\hline \multicolumn{7}{|l|}{ Malaria control } \\
\hline Use of a bednet & & & & & & \\
\hline No use & 1002 & 49.9 & 1297 & 62.6 & 317.571 & $<0.0001$ \\
\hline$<1 \mathrm{yr}$ & 940 & 46.8 & 487 & 23.5 & & \\
\hline $1-2$ yrs & 68 & 3.4 & 289 & 13.9 & & \\
\hline IRS past 6 months & & & & & & \\
\hline Yes & 1140 & 56.7 & 759 & 36.6 & 165.854 & $<0.0001$ \\
\hline No & 869 & 43.3 & 1313 & 63.3 & & \\
\hline Fly screen & & & & & & \\
\hline Yes & 376 & 18.8 & 408 & 19.7 & 0.565 & 0.452 \\
\hline No & 1627 & 81.2 & 1663 & 80.3 & & \\
\hline
\end{tabular}


Table 1 Characteristics of study participants (Continued)

\begin{tabular}{|c|c|c|c|c|c|c|}
\hline \multirow[t]{2}{*}{ Variable } & \multicolumn{2}{|l|}{ CS1 } & \multicolumn{2}{|l|}{ CS2 } & \multirow[t]{2}{*}{$x^{2}$} & \multirow[t]{2}{*}{$P$-value } \\
\hline & $n$ & $\%$ & $n$ & $\%$ & & \\
\hline \multicolumn{7}{|l|}{ Malaria morbidity } \\
\hline \multicolumn{7}{|l|}{ Previous episodes } \\
\hline 0 & 587 & 29.3 & 606 & 29.3 & & \\
\hline $1-3$ & 656 & 32.8 & 614 & 29.6 & 6.479 & 0.091 \\
\hline $4-10$ & 455 & 22.7 & 487 & 23.5 & & \\
\hline$>10$ & 303 & 15.1 & 359 & 17.3 & & \\
\hline \multicolumn{7}{|l|}{ Episode in past two weeks } \\
\hline Yes & 24 & 1.2 & 21 & 1.0 & 0.307 & 0.580 \\
\hline No & 1986 & 98.8 & 2052 & 99.0 & & \\
\hline \multicolumn{7}{|l|}{ Current symptoms } \\
\hline Fever $^{a}$ & 108 & 5.4 & 84 & 4.1 & 3.974 & 0.046 \\
\hline Symptoms other than fever ${ }^{b}$ & 76 & 3.8 & 16 & 0.8 & 42.857 & $<0.0001$ \\
\hline
\end{tabular}

than for CS2 (Table 1, $P<0.0001$ ). In addition, considering data from both CS1 and CS2, 19.2\% (784/4083) of participants lived in households with screened windows.

\section{Plasmodium spp. prevalence}

In CS1, 86 of 2010 (4.3\%) participants were infected with $P$. vivax as determined by qPCR. Prevalence by qPCR was thus 2.7-fold higher than prevalence by LM (1.6\%) (Table 2). Fifty percent (43/86) of all $P$. vivax infected individuals (qPCR) carried $P$. vivax gametocytes when detected by RT-qPCR, whereas $56.3 \%(18 / 32)$ of $P$. vivax infections detected by LM were positive for gametocytes. A modestly lower prevalence of $P$. vivax infections was observed in CS2 with $3.4 \%(70 / 2,073)$ of participants having qPCR-detectable infections, although this difference between surveys did not reach statistical significance, irrespective of considering all participants (Table 2, $\left.\chi^{2}=2.259, d f=1, P=0.133\right)$ or only those that were surveyed twice (CS1: 45/963, 4.7\% vs CS2: 33/963, $\left.3.4 \%, \quad X^{2}=1.924, \quad d f=1, \quad P=0.165\right) . \quad$ A comparable number of $P$. vivax infections were gametocyte positive in both surveys $\left(50.0 \%\right.$ vs $42.9 \%, X^{2}=0.791$, $d f=1, P=0.374)$. Total $P$. vivax parasitemia was comparable in both surveys (median gene copy number/ $\mu$ l 21.8 vs 35.0, Mann-Whitney test: $U=2643.5, n_{1}=$ $86, n_{2}=70, P=0.192$ ) but $p v s 25$ transcript numbers were significantly higher in gametocyte positive samples in CS2 compared to CS1 (median 11.4 vs 44.6 transcripts/ $\mu \mathrm{l}$, Mann-Whitney test: $U=417.5, n_{1}=$ $\left.43,=n_{2}=30, P=0.011\right)$. Overall, $P$. falciparum infections were rare (Table 2): In CS1 only 15 mono $P$. falciparum infections were detected by qPCR (0.8\%); in CS2 a single mixed $P$. falciparum/P. vivax infection was detected $\left(0.05 \%, X^{2}=12.739, d f=1, P=0.0004\right)$. Among $P$. falciparum positive samples in CS1, 81.8\% (9/11) showed presence of gametocytes by light microscopy and $93.3 \%(14 / 15)$ by qPCR. In CS2, the only $P$. falciparum positive sample was also positive for gametocytes by qRT-PCR.

Table 2 Prevalence of infection by P. vivax (Pv total), P. vivax gametocytes (Pv gam), P. falciparum (Pf total) and P. falciparum gametocytes (Pf gam) as determined by light microscopy and PCR. Microscopy data available only for cross-sectional survey (CS) 1, overall prevalence by PCR determined as $18 \mathrm{~S}$ rDNA copies/ $\mu$ l by qPCR, gametocyte prevalence by PCR determined by RT-qPCR (pvs25 transcripts/ $\mu l)$

\begin{tabular}{|c|c|c|c|c|c|c|c|c|c|c|c|c|}
\hline & \multicolumn{4}{|c|}{ CS1 Microscopy $(n=2010)$} & \multicolumn{4}{|c|}{ CS1 PCR $(n=2010)$} & \multicolumn{4}{|c|}{ CS2 PCR $(n=2073)$} \\
\hline & $n$ & $\%$ & Median paras./Ml & IQR & $n$ & $\%$ & Median cop./transcr./ul & IQR & $n$ & $\%$ & Median cop./transcr./ul & IQR \\
\hline Pv total & 32 & 1.8 & 195.0 & $60.0-555.0$ & 86 & 4.3 & 21.8 & $6.0-246.4$ & $70^{a}$ & 3.4 & 35.0 & $12.3-273.0$ \\
\hline Pv gam & $18^{\mathrm{b}}$ & 1.0 & 60.0 & $30.0-60.0$ & 43 & 2.1 & 11.4 & $3.8-85.1$ & $30^{\mathrm{a}}$ & 1.4 & 44.6 & $13.5-175.1$ \\
\hline Pf total & 11 & 0.5 & 240.0 & $90.0-600.0$ & 15 & 0.8 & 151.8 & $8.1-681.3$ & $1^{a}$ & 0.05 & 5.1 & - \\
\hline Pf gam & $9^{b}$ & 0.4 & 150.0 & $60.0-270.0$ & 14 & 0.7 & 434.9 & $40.0-1272.8$ & $1^{\mathrm{a}}$ & 0.05 & 154.8 & - \\
\hline
\end{tabular}

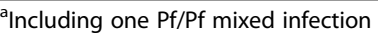

${ }^{\mathrm{b}}$ One slide was negative for asexual blood stages 
Morbidity and univariate risk-factors for $P$. vivax infection Combining the results of both CS1 and CS2, prevalence of $P$. vivax was about twice as high in Brasileirinho and Puraquequara $(4.3 \%)$ than in Ipiranga $\left(2.1 \%, \chi^{2}=10.198\right.$, $d f=2, P=0.006$ ) (Additional file 4: Table S4). The proportion of infected individuals was higher among male study participants $(4.8 \%)$ than among female participants (2.7\%) and male participants had a significantly higher risk for infection (OR: 1.81, 95\% CI: 1.28-2.58, $\chi^{2}=$ $12.137, d f=1, P=0.0005)$. Adults $(\geq 18$ years, prevalence $4.8 \%$ ) had a higher risk for infection than children (2.4\%) (OR: 2.02, 95\% CI: 1.40-2.97, $X^{2}=$ 15.369, $d f=1, P=0.0001)$. The risk for infection was significantly higher for workers from the agriculture sector than for office workers and individuals pursuing higher education (OR: 1.81, 95\% CI: 1.03-3.17, $\chi^{2}$ $=28.208, d f=1, P=0.0001$ ).

Prevalence amongst new residents (4.1\%) was only slightly higher than that for individuals that lived in the study area for more than two months $(3.8 \%$, OR: 1.08, 95\% CI: 0.42-2.32, $\chi^{2}=0.035, d f=1, P=0.852$ ). Individuals with recent travel history showed a lower prevalence $(2.4 \%)$ than individuals that did not leave the study area (4.0\%, OR: 0.60, 95\% CI: $0.30-1.18, \chi^{2}$ $=2.271, d f=1, P=0.132$. Plasmodium vivax prevalence was higher amongst individuals using LLINs (4.7\%, OR: $1.53,95 \%$ CI: $1.11-2.11, \chi^{2}=6.796, d f=1$,
$P=0.009$ ) and for participants living in IRS-treated houses $\left(4.6 \%\right.$, OR: $1.51,95 \%$ CI: $1.09-2.09, \chi^{2}=6.361$, $d f=1, P=0.012)$ as compared to persons not using either intervention measure $(3.1 \%)$. The proportion of $P$. vivax infected individuals was lower, but not significant, if the individual's home had screened windows (3.2\%), compared to those individuals living in houses with unscreened windows (4.0\%, OR: 0.80, 95\% CI: $0.52-1.24, \chi^{2}$ $=1.006, d f=1, P=0.316$ ).

Study participants that had not experienced malaria episodes during their lifetime showed a lower risk for infection (prevalence $0.8 \%$ ) than participants with one or more previous malaria infections $(5.1 \%$, OR: 7.04 , 95\% CI: 3.59-15.75, $\left.X^{2}=43.030, d f=1, P<0.0001\right)$. Furthermore, $P$. vivax prevalence was higher in individuals that experienced a recent malaria episode $(7.0 \%)$ than in individuals with no recent episode (3.8\%, OR: 1.94, 95\% CI: 0.77-4.88, Fisher's exact test $P=0.195$ ). Individuals presenting fever the day of the visit or during the past $48 \mathrm{~h}$ had a significantly increased risk for infection by $P$. vivax (prevalence 14.1\%, OR: 4.77, 95\% CI: $\left.3.06-7.43, \chi^{2}=57.510, d f=1, P<0.0001\right)$. Individuals with symptoms other than fever (up to $48 \mathrm{~h}$ before visit) had a moderately increased, albeit not-significant, prevalence $(5.4 \%)$ as compared to asymptomatic individuals (3.63\%, OR: 1.55 , 95\% CI: 0.48-34.83, Fisher's exact test: $P=0.386$ ) (Additional file 4: Table S4).

Table 3 Multivariate predictors of infection by $P$. vivax

\begin{tabular}{|c|c|c|c|c|c|}
\hline & $n$ & $\%$ & AOR & $95 \% \mathrm{Cl}$ & $P$-value \\
\hline \multicolumn{6}{|l|}{ Gender } \\
\hline Male & 2138 & 4.8 & 2.22 & $1.41-3.51$ & 0.001 \\
\hline Female & 1945 & 2.7 & - & - & \\
\hline \multicolumn{6}{|l|}{ Occupation } \\
\hline Office worker and higher education student & 614 & 3.3 & - & - & \\
\hline Agriculture/fishing & 626 & 5.8 & 1.68 & $0.96-2.95$ & 0.009 \\
\hline Housewife & 635 & 4.3 & 2.46 & $1.28-4.71$ & \\
\hline Infants and preschool children & 612 & 2.0 & 1.47 & $0.63-3.40$ & \\
\hline School children & 965 & 2.5 & 0.95 & $0.51-1.78$ & \\
\hline Retired & 203 & 3.9 & 1.12 & $0.48-2.63$ & \\
\hline Unemployed/other & 423 & 6.9 & 2.13 & $1.16-3.89$ & \\
\hline \multicolumn{6}{|l|}{ Number of previous episodes } \\
\hline 0 & 1193 & 0.8 & - & & \\
\hline $1-3$ & 1270 & 3.9 & 5.25 & $2.37-11.65$ & $<0.001$ \\
\hline $4-10$ & 942 & 5.9 & 7.75 & $3.46-17.36$ & \\
\hline$>10$ & 662 & 6.2 & 7.01 & $2.95-16.64$ & \\
\hline \multicolumn{6}{|l|}{ Fever $^{a}$} \\
\hline Yes & 192 & 14.1 & 4.66 & $2.88-7.53$ & $<0.001$ \\
\hline No & 3891 & 3.3 & - & - & - \\
\hline
\end{tabular}

${ }^{\mathrm{a}}>37.5^{\circ} \mathrm{C}$ at visit or past $48 \mathrm{~h}$ 
Multivariate risk factors for $P$. vivax infection

After adjusting for potential confounders (community, vector control activities and age) gender, occupation, history of malaria and fever remained as independent predictors for $P$. vivax infection. In the multivariate analysis male individuals were at higher risk for infection by $P$. vivax than female individuals (Table 3, AOR: 2.22, 95\% CI: $1.41-3.51, P=0.001)$. Individuals that reported to have had one or more previous malaria episodes in their lives were at much higher risk for $P$. vivax infection than individuals that had no previous infection (Table 3, $P<0.001)$. Another factor strongly associated with $P$. vivax infection was fever (during visit or during the past 48 h) (AOR: 4.66, 95\% CI: 2.88-7.53, $P<0.001$ ).

\section{Carriage of $P$. vivax gametocytes}

Overall, about half of all $P$. vivax infected individuals (73/ $156,46.8 \%)$ were positive for gametocytes, detected by RT-qPCR. Both in the univariate (OR: 2.73, 95\% CI: 1.854.05, $P<0.0001$ ) and in the multivariate (AOR: $2.67,95 \%$ CI: $1.90-3.77, P<0.0001)$ analyses the only risk factor associated with detectable carriage of gametocytes was $P$. vivax parasite density. We modelled the probability for carriage of gametocytes (defined as positivity by RT-qPCR in a $50 \mu$ l blood sample) depending on $P$. vivax density using a generalised additive model (Fig. 2a). For low parasite densities $(<1018 S$ rDNA copies/ $\mu \mathrm{l})$ the probability was below $30.0 \%$ and increased only slightly. At 100 copies/ $\mu$ l the probability to carry gametocytes was $50.0 \%$ and increased dramatically reaching a probability of $90.0 \%$ at 10,000 copies/ $\mu \mathrm{l}$. Gametocyte density (measured as pvs 25 transcripts $/ \mu \mathrm{l})$ also increased with $P$. vivax density $\left(r^{2}=\right.$ 0.496, $P<0.0001$ ) (Fig. 2b).

Both prevalence of $P$. vivax blood-stages and prevalence of $P$. vivax gametocytes increased with age. The density of $P$. vivax blood-stages and pvs 25 transcripts was highest in children younger than 12 years (Fig. 3).

\section{Asymptomatic and submicroscopic infections}

Overall, 82.7\% (129/156) of all people infected with $P$. vivax did not report a concurrent febrile illness and $75.0 \%$ $(117 / 156)$ of infected individuals did not report fever or any other symptoms (the day of visit or up to $48 \mathrm{~h}$ before) nor did they report with symptoms to a malaria clinic during the 30 days before visit. Of these asymptomatic individuals, $11.1 \%$ (13/117) developed symptoms within 30 days after the visit. Thus, $67.3 \%$ (104/156) of all $P$. vivax infected individuals were and remained asymptomatic. There was no significant difference between CS1 $(56 / 86)$ and CS2 (48/70) $\left(\chi^{2}=0.2073, d f=1, P=0.649\right)$. Similarly, $76.7 \%(56 / 73)$ of $P$. vivax gametocyte positive individuals were afebrile and 64.4\% (47/73) were asymptomatic. $53.4 \%(39 / 73)$ of individuals carrying $P$. vivax gametocytes were asymptomatic (at visit and past 30 days) and did not develop any symptoms within 30 days after the visit (Fig. 4). Febrile (at visit or past 48 h) P. vivax infections were of significantly higher density (median copy numbers/ $\mu \mathrm{l}$ : 463.8, IQR: 13.5-5030.4) than afebrile infections (20.8, IQR: 6.7-113.5, Mann-Whitney test: $U=$ 985.5, $\left.n_{1}=27, n_{2}=129, P=0.0004\right)$. Individuals showing any malaria-related symptoms up to the past $48 \mathrm{~h}$ also exhibited a significantly higher parasite density (median copy numbers: 282.6, IQR: 6.9-4452.6) as compared to asymptomatic infections (20.3, IQR: 6.7-103.4, $P=0.0007$, Fig. 5). In CS1, 65.6\% (42/64) of asymptomatic and $54.5 \%(12 / 22)$ of symptomatic $P$. vivax infections were submicroscopic $\left(\chi^{2}=0.860, d f=1\right.$, $P=0.354$ ) (Fig. 6).

Of the $16 P$. falciparum infections (including 1 mixed infection), 12 (75.0\%) were asymptomatic (at visit or up
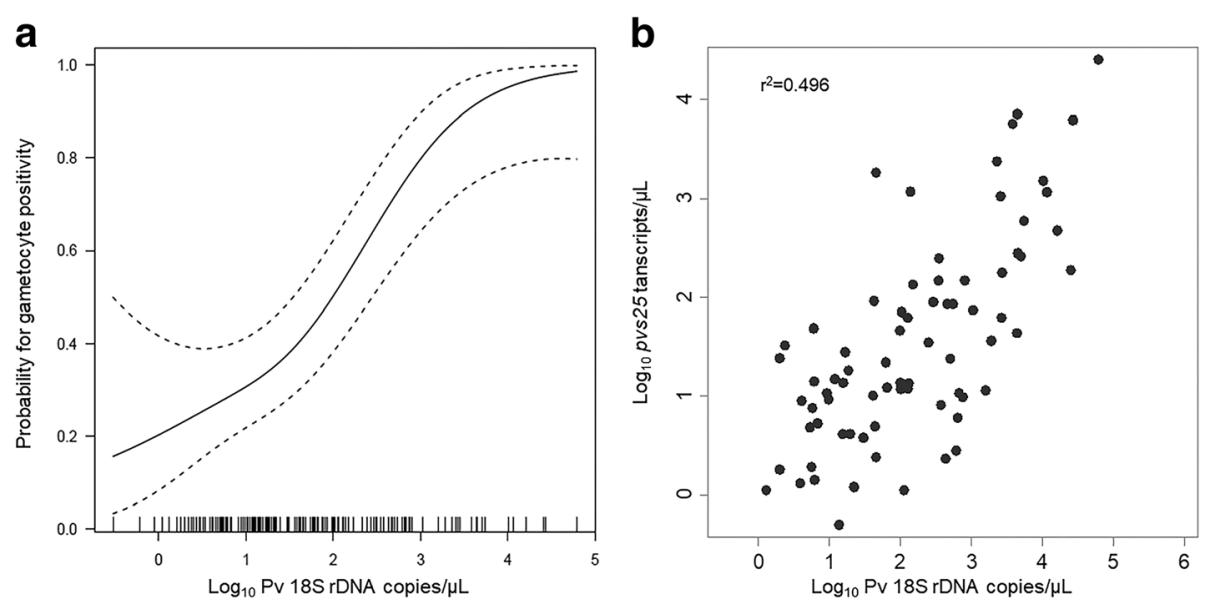

Fig. 2 Relation between P. vivax parasitaemia and gametocyte carriage and gametocyte density. A generalized additive model was used to describe gametocyte positivity (a). Plasmodium vivax parasitaemia was determined by qPCR (18S rDNA copies/ $\mu$ l) and gametocyte density by RT-qPCR (pvs25 transcripts/ $\mu l)(\mathbf{b})$ 

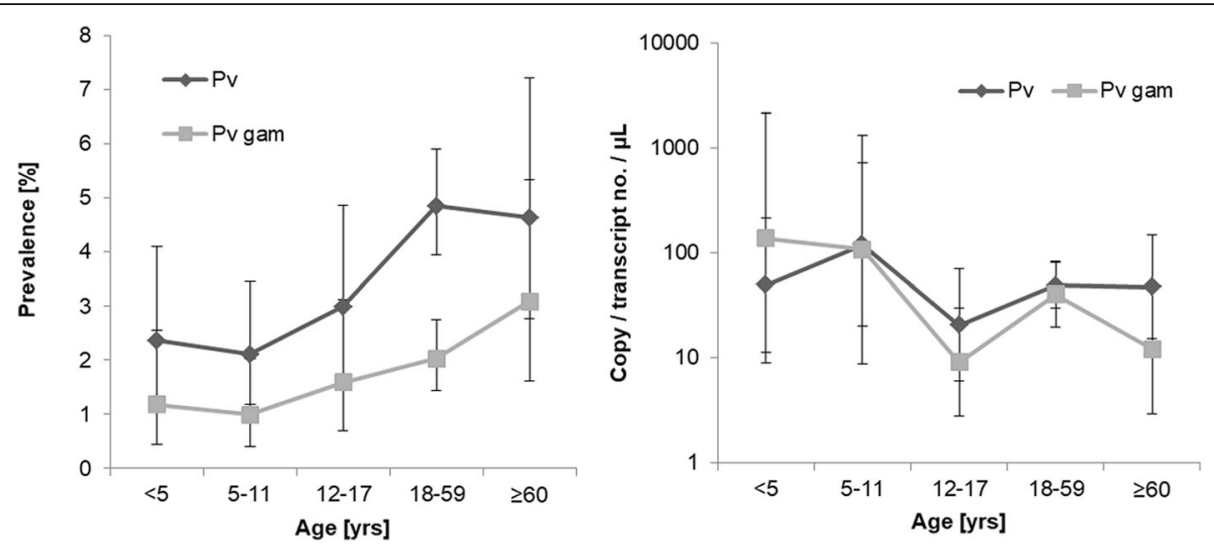

Fig. 3 Prevalence of $P$. vivax blood-stages and P. vivax gametocytes and parasite densities in different age groups. Geometric mean and $95 \%$ confidence interval are shown Plasmodium vivax blood-stage (Pv) density was determined by qPCR (18S rDNA copies/ $\mu$ l) and gametocyte (Pv gam) density by RT-qPCR (pvs25 transcripts/ $\mu$ l)

to $48 \mathrm{~h}$ before). Of the 15 individuals carrying P. falciparum gametocytes, 11 (73.3\%) were asymptomatic. None of the $P$. falciparum infected individuals developed symptoms within 30 days after the visit (Fig. 4). Four (35.7\%) asymptomatic P. falciparum infections in CS1 were submicroscopic, while all symptomatic infections were detectable by LM (Fig. 6).

\section{Discussion}

The two cross-sectional surveys conducted in the periphery of Manaus confirmed the low prevalence of malarial infections in the area. This is in accordance with the significant successes recently achieved in Brazil regarding malaria control, whereby from 2000 to 2011 the number of cases has been more than halved [24]. This success has been attributed to classical intervention measures including early recognition and treatment of malaria cases and a high coverage of LLIN distribution
[2]. The prevalence observed in CS2 (August-September 2013) was slightly lower than that of CS1 (NovemberDecember 2012). Although in the Amazon region malaria transmission usually peaks during the dry period from June to September, year-to-year fluctuations occur due to variation in climatic conditions $[6,17,25]$.

Most infections were caused by P. vivax; only $14.9 \%$ (CS1) and $1.4 \%$ (CS2), respectively, were caused by P. falciparum, in accordance with a decreasing trend in P. falciparum cases in Brazil since 1989 [25]. In the municipality of Manaus, for instance, P. falciparum infections dropped from $40.8 \%$ in 1986 to $6.5 \%$ in 2003 [7]. In addition to intervention strategies focusing on early diagnosis and treatment, the introduction of artemisinin-based combination therapy in 2006 further enhanced this trend [2]. Early treatment is generally more efficient against $P$. falciparum than $P$. vivax [26], as the appearance of mature

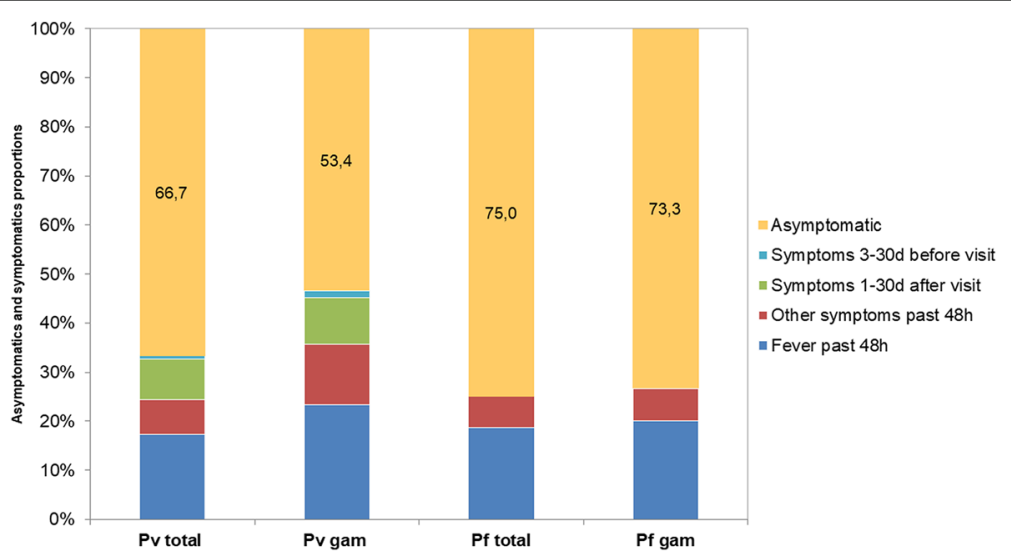

Fig. 4 Proportion of asymptomatic infections. Fever and other symptoms as reported on day of visit or up to $48 \mathrm{~h}$ before. Symptoms more than 2 days before or after visit according to Information System for Epidemiological Surveillance of Malaria (SIVEP-Malária). Abbreviations: Pv total, all P. vivax blood stages; Pv gam, P. vivax gametocytes; Pf total, all P. falciparum blood stages; Pf gam, P. falciparum gametocytes 


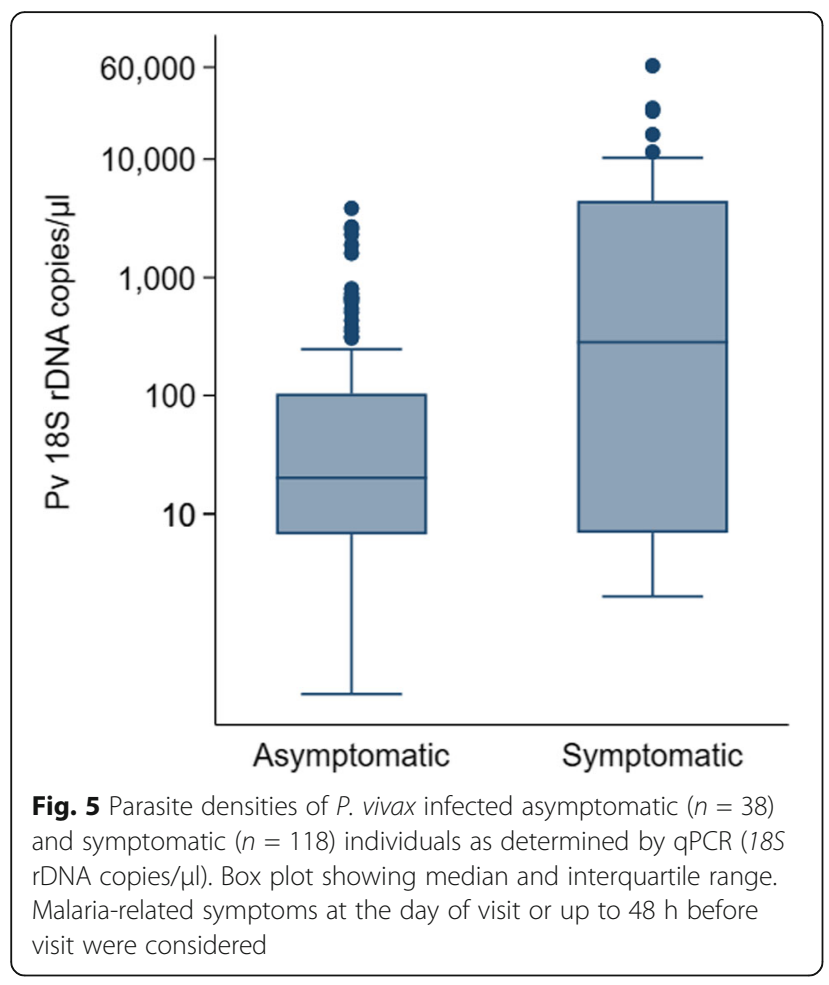

gametocytes occurs later during infection. Additionally, the need to ensure radical treatment of $P$. vivax often jeopardizes the adequate management of this species because of compliance issues. As a consequence, in many co-endemic settings successful control has resulted in a shift from $P$. falciparum to $P$. vivax being the most prevalent parasite $[27,28]$.

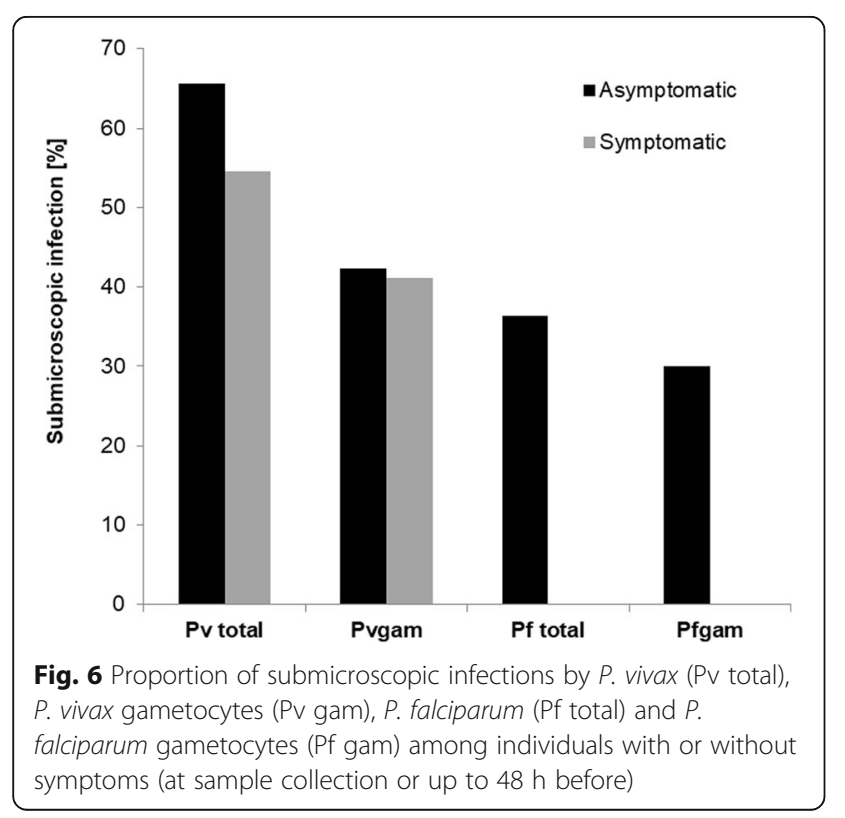

Multivariate analysis revealed gender and occupation as key factors associated with infection by $P$. vivax, with male individuals being at higher risk as compared to female. This difference can possibly be explained by different occupation and leisure related behaviour. For instance, one third of adult men were working in the agricultural sector, which was associated with an elevated risk for infection (although not significant). One fifth of adult men had stated to be unemployed or pursue other not classified occupations. That group was significantly associated with infection. Among the different occupation groups housewives were at highest risk for $P$. vivax infection, probably because many houses in the study area include an outside area where activities such as laundry or gardening are performed. Our observations are in agreement with a previously published study conducted in a farming settlement in the South of the Amazon State where an increased risk of infection for individuals working in agriculture or housekeeping occurred [10]. In the univariate model age also represented a risk factor for $P$. vivax infections, showing an elevated risk for adolescents and adults, although this is likely associated with behaviour rather than age. The strongest predictor for $P$. vivax infection was fever. However, one has to keep in mind that in areas with such high proportion of asymptomatically infected individuals, in some cases the fever potentially might be caused by other concurrent (often unrelated) infectious diseases.

We were not able to find a protective effect for LLINs or IRS. Univariate analyses showed instead that both intervention measures were associated with a higher risk for infection by $P$. vivax. This can probably be explained by increased attention and control measures by both health post agents and inhabitants in known transmission hotspots. Whether LLINs and IRS can reduce malaria transmission in South America is not fully clear yet, as the main vector in the region, An. darlingi, is to a large extent exophilic, with its main peak of activity occurring at nightfall [29]. In addition, many of the detected $P$. vivax infections might result from reactivated hypnozoites rather than from recent mosquito bites. According to a study carried out in Papua New Guinea, relapses may be the cause of about $80 \%$ of $P$. vivax episodes [30]. A recent study from the Brazilian Amazon also suggests a significant contribution of relapses in this region [14].

Amongst individuals positive for $P$. vivax, we identified $P$. vivax blood-stage parasite density as the only risk factor for carrying gametocytes. Other studies also identified (asexual) blood stage density as a major predictor for carriage of gametocytes [31, 32]. Furthermore, we observed a weak correlation between total parasitaemia and gametocyte density. In $P$. vivax, gametocytes appear early in infection and their production continues 
throughout the infection, thus explaining increased risk for gametocyte carriage and increasing gametocyte densities with increasing $P$. vivax parasitaemia [33].

About half of all $P$. vivax positive individuals were positive for gametocytes. Other reports show higher rates of gametocyte carriers; however, these symptomatic individuals had high parasite densities that correlated with better detection of gametocytes [10, 34, 35]. A study that used large blood volumes for RNA extraction and subsequent RNA extraction achieved a high sensitivity of detecting both asexual and sexual stages [10], while in our study only $50 \mu \mathrm{l}$ of patient blood was used for RNA extraction. In addition, the proportion of gametocyte positive individuals depends on the sensitivity of the assay detecting parasitaemia of blood-stage parasites - the more sensitive, the lower the parasite densities being detected, and therefore the probability of being positive for (detectable) gametocytes [32]. Gametocyte count by LM was performed on 200 leukocytes in the samples from this study, which may have underestimated the prevalence of gametocytes in infected individuals. The sensitivity of gametocyte detection could be improved counting more microscopic fields.

Up to three quarters of all $P$. vivax infected individuals appeared to be free of symptoms on the day of blood sampling. The majority of them also remained free of symptoms for the following 30 days, as previously reported $[10,15]$. We were able to demonstrate a high proportion of asymptomatic infections in a low transmission setting, as previously described in the Americas [15, 36].

Among the individuals positive for $P$. vivax gametocytes, the proportion of asymptomatic individuals was smaller. This can be explained by the lower chance to detect gametocytes in low blood-stage parasite densities, such as observed in asymptomatic individuals. Despite having lower parasitemia, and therefore lower gametocytemia, asymptomatic infections had been shown to be able to infect mosquitoes [37] and hence, might be an important reservoir, especially for seasonal outbreaks [26]. A study in southern Amazon State estimated that $54.4 \%$ of all parasite biomass belonged to asymptomatic infected individuals, accounting for $56.6 \%$ of all infections [14]. As asymptomatic carriers do not seek healthcare facilities, they do not receive treatment and therefore might remain infective over long periods. Furthermore, a large proportion of asymptomatic infections is submicroscopic and would therefore by standard diagnostic methods remain undetected. Overall, more than half of $P$. vivax infections (62.8\%) were submicroscopic, as opposed to only $26.7 \%$ of $P$. falciparum infections. Two recent reviews reported similar proportions of subpatent $(69.5 \%$ [38] and 67.0\% [39]) P. vivax infections and substantially lower proportions of subpatent $P$. falciparum infections in various studies from different continents. In Brazil only LM positive infections are treated, thus the malaria subpatent infections remain untreated.

This work is the first large-scale survey assessing the infectious reservoir, and therefore the malaria transmission potential, with molecular methods in the peri-urban area of Manaus. We identified a large proportion of asymptomatic and submicroscopic malaria infections implicating that these might play an important role in malaria transmission in the study area. These infections are not only a burden to the local population, but also responsible for infections in (malaria-naïve) visitors [6]. Classical intervention measures are not effective against subpatent infections and new control measures will therefore be needed to achieve elimination. Although PCR is not suitable for routine diagnostics, as it is expensive and technically challenging, molecular data as those arising from our study can provide important information on the infectious reservoir, for instance for mathematical models [39]. A clearer understanding of the dynamics of the infectious reservoir over time is needed and longitudinal studies will be critical in order to assess the duration of asymptomatic infections and their likely role in sustaining transmission in the area.

\section{Conclusions}

This study shows a substantial proportion of asymptomatic and submicroscopic $P$. vivax infections in the studied area. The majority of gametocyte carriers did not present symptoms, thus indicating that asymptomatic infections might significantly contribute to malaria transmission in this region. Therefore, efficient control and malaria elimination tools need to include approaches also targeting the asymptomatic reservoir. Longitudinal studies are needed in these locations to analyze the incidence of malaria infection and the risk factors for $P$. vivax gametocyte carriage.

\section{Additional files}

\section{Additional file 1: Table S1. Primers and probes used for amplification of $18 \mathrm{~S}$ rRNA, pvs 25 and pfs 25 , using qPCR or RT-qPCR assays including reaction efficiency and detection limits. (DOCX $16 \mathrm{~kb}$ ) \\ Additional file 2: Table S2. Reaction mixes for amplification of 185 rRNA, pvs 25 and pfs 25 , using qPCR or RT-qPCR. The table describes the reagents, concentrations and template volume used in the $\mathrm{PPCR}$ and RT-qPCR assays. (DOCX $13 \mathrm{~kb}$ ) \\ Additional file 3: Table S3. Conditions for amplification of 185 rRNA, pvs25 and pfs25, using qPCR or RT-qPCR assays. (DOCX $14 \mathrm{~kb}$ ) \\ Additional file 4: Table S4. Univariate analysis for predictors of $P$. vivax infection. (DOCX $22 \mathrm{~kb}$ )}

\section{Abbreviations}

AOR: Adjusted OR; Cl 95\%: 95\% confidence onterval; CONEP: Brazilian National Committee of Ethics; CS: Cross-sectional; EDTA: Ethylenediaminetetraacetic 
acid; FMT-HVD: Fundação de Medicina Tropical Dr Heitor Vieira Dourado; IQR: Interquartile range; IRS: Indoor residual spraying; LLINs: Long-lasting insecticidetreated nets; LM: Light microscopy; OR: Odds ratio; PBS: Phosphate buffered saline; PCR: Polymerase chain reaction; Pf gam: P. falciparum gametocytes; Pf: P. falciparum blood-stage; Pv gam: P. vivax gametocytes; Pv: P. vivax blood-stage; qPCR: Quantitative PCR; RBC: Red blood cells; RT-qPCR: Quantitative reverse transcription PCR; SIVEP-Malaria: System for Epidemiological Surveillance of Malaria

\section{Acknowledgements}

Above all, we would like to thank the members of the three communities that participated in our study. We thank all the health post staff from the Brazilian Health Surveillance Agency (SVS) for their support during the field work. We thank all staff from the Malaria Department of FMT-HVD, especially the head of department Monica Costa and the microscopy team. We thank Rahel Wampfler, Natalie Hofmann, Cristian Koepfli and Andreea Waltmann for their advice on field and laboratory work and Connie SN Li Wai Suen for statistical support.

\section{Funding}

This work was supported by a grant from Bill \& Melinda Gates Foundation to the TransEPI consortium. Additional support was provides by Fundação de Amparo à Pesquisa do Estado do Amazonas (FAPEAM), Brazil and NHMRC (\#1043345). IM is supported by an NHMRC Senior Research Fellowship.

\section{Availability of data and materials}

All data generated or analysed during this study are included in this published article and its Additional files 1, 2, 3 and 4. Although the datasets generated and/or analysed during the current study are not publicly available, as the information in the databases will be used in future publications, they are available from the corresponding author upon reasonable request.

\section{Authors' contributions}

Conceived and designed the experiments: IM, IF, MVGL, QB, WMM and WPT. Prepared and performed field work: ACGA, LWB, AK, SVS, AJMC, MAB and EFGF. Sample processing: EFGF, KMMC, ACGA, AJMC and AK. Performed the experiments: AK, ACGA and AJMC. Data entry and analyses: AK, ACGA, AJMC, VSS and IM. Wrote the paper: AK, ACGA, AJMC, QB, IM and WMM. All authors read and approved the final manuscript.

\section{Ethics approval and consent to participate}

This study was approved by the Brazilian National Committee of Ethics (CONEP) (349.211/2013) and by the Committee of Ethics for Clinical Investigation from Barcelona Hospital Clinic (7306/2012). All participants were informed about the objectives of the study as well as potential risks and benefits of their participation in the study. An informed consent form was signed by all study participants or by a parent or legal guardian in case of participants younger than 18 years. Children between 12-17 years signed an additional assent form

\section{Consent for publication}

Not applicable.

\section{Competing interests}

The authors declare that they have no competing interests.

\section{Publisher's Note}

Springer Nature remains neutral with regard to jurisdictional claims in published maps and institutional affiliations.

\footnotetext{
Author details

${ }^{1}$ Fundação de Medicina Tropical Dr. Heitor Vieira Dourado (FMT-HVD), Av. Pedro Teixeira, N.25, Dom Pedro, Manaus, Amazonas CEP: 69040-000, Brazil. 2Universidade do Estado do Amazonas (UEA), Av. Djalma Batista, N. 3578, Flores, Manaus, Amazonas CEP: 69005-010, Brazil. ${ }^{3}$ ISGlobal, Barcelona Ctr. Int. Health Res. (CRESIB), Hospital Clínic - Universitat de Barcelona, Carrer del Rosselló, 132, 08036 Barcelona, Spain. ${ }^{4}$ Instituto Nacional de Pesquisas da Amazônia (INPA), Av. André Araújo, N. 2.936, Petrópolis, Manaus CEP: 69067-375, Brazil. 'Fundação de Vigilância em Saúde do Amazonas, Sala de Análise de Situação em Saúde, Av. Torquato Tapajós, N. 6132, Colônia Santo Antonio, Manaus CEP:69093-018, Brazil. ${ }^{6}$ Centro de Investigação em Saúde de Manhiça (CISM), Rua 12, Cambeve, Vila de Manhiça, CP 1929 Maputo, Mozambique. ${ }^{7}$ Institució Catalana de Recerca i Estudis Avançats (ICREA),
}

Passeig Lluís Companys, 2308010 Barcelona, Spain. ${ }^{8}$ Swiss Tropical and Public Health Institute, Socinstrasse 57, 4051 Basel, Switzerland. ${ }^{9}$ University of Basel, Petersplatz 1, 4001 Basel, Switzerland. ${ }^{10}$ Walter and Eliza Hall Institute, Parkville, Australia. ${ }^{11}$ Instituto de Pesquisas Leônidas e Maria Deane (ILMD), Manaus, Brazil.

Received: 3 November 2017 Accepted: 7 March 2018

Published online: 20 March 2018

\section{References}

1. World Health Organization. World Malaria Report 2017. Geneva: WHO Press; 2017.

2. Oliveira-Ferreira J, Lacerda MVG, Brasil P, Ladislau JLB, Tauil PL, Daniel-ribeiro CT. Malaria in Brazil: an overview. Malar J. 2010:9:115.

3. Siqueira AM, Mesones-Lapouble O, Marchesini P, Sampaio VS, Brasil P, Tauil $\mathrm{PL}$, et al. Plasmodium vivax landscape in Brazil: scenario and challenges. Am J Trop Med Hyg. 2016;95:87-96.

4. Griffing SM, Tauil PL, Udhayakumar V, Silva-Flannery L. A historical perspective on malaria control in Brazil. Mem Inst Oswaldo Cruz. 2015;110: 701-18.

5. da Silva-Nunes M, Moreno M, Conn JE, Gamboa D, Abeles S, Vinetz JM, et al. Amazonian malaria: asymptomatic human reservoirs, diagnostic challenges, environmentally driven changes in mosquito vector populations, and the mandate for sustainable control strategies. Acta Trop. 2012;121:281-91.

6. Sampaio VS, Siqueira AM, Alecrim MGC, Mourão MPG, Marchesini PB, Albuquerque BC, et al. Malaria in the State of Amazonas: a typical Brazilian tropical disease influenced by waves of economic development. Rev Soc Bras Med Trop. 2015;48:4-11

7. Saraiva MDGG, Amorim RDS, Moura MAS, Martinez-Espinosa FE, Barbosa MDGV. Urban expansion and spatial distribution of malaria in the municipality of Manaus, State of Amazonas. Rev Soc Bras Med.Trop. 2009;42: $515-22$

8. Martins-Campos KM, Pinheiro WD, Vitor-Silva S, Siqueira AM, Melo GC, Rodrigues IC, et al. Integrated vector management targeting Anopheles darlingi populations decreases malaria incidence in an unstable transmission area, in the rural Brazilian Amazon. Malar J. 2012;11:351.

9. Zimmerman $\mathrm{RH}$. Ecology of malaria vectors in the Americas and future direction. Mem Inst Oswaldo Cruz. 1992;87:371-83.

10. Barbosa S, Gozze AB, Lima NF, Batista CL, Bastos MDS, Nicolete VC, et al. Epidemiology of disappearing Plasmodium vivax malaria: a case study in rural Amazonia. PLoS Negl Trop Dis. 2014;8:e3109.

11. da Silva NS, da Silva-Nunes M, Malafronte RS, Menezes MJ, D'Arcadia RR, Komatsu NT, et al. Epidemiology and control of frontier malaria in Brazil: lessons from community-based studies in rural Amazonia. Trans R Soc Trop Med Hyg. 2010;104:343-0.

12. Katsuragawa TH, Gil LHS, Tada MS, de Almeida e Silva A, JDAN C, MDS A, et al. The dynamics of transmission and spatial distribution of malaria in riverside areas of Porto Velho, Rondônia, in the Amazon region of Brazil. Plos One. 2010:5:e9245.

13. Ladeia-Andrade S, Ferreira MU, Carvalho MED, Curado I. Age-dependent acquisition of protective immunity to malaria in riverine populations of the Amazon Basin of Brazil. Am J Trop Med Hyg. 2009;80:452-9.

14. Vitor-Silva S, Siqueira AM, de Souza SV, Guinovart C, Reyes-Lecca RC, de Melo GC, et al. Declining malaria transmission in rural Amazon: changing epidemiology and challenges to achieve elimination. Malar J. 2016:15:266.

15. Suárez-Mutis MC, Cuervo P, FMS L, Moraes-Avila SL, Ferreira AW, Fernandes $\mathrm{O}$, et al. Cross sectional study reveals a high percentage of asymptomatic Plasmodium vivax infection in the Amazon Rio Negro area, Brazil. Rev Inst Med Trop. 2007:49:159-64

16. Instituto Brasileiro de Geografia e Estatística. Brazilian Demographic Census. Brazil; 2016. https://cidades.ibge.gov.br/xtras/perfil.php?lang=\&codmun= 130260\&search=amazonas/manaus. Accessed 23 Sept 2017.

17. Wolfarth BR, Filizola N, Tadei WP, Durieux L. Epidemiological analysis of malaria and its relationships with hydrological variables in four municipalities of the State of Amazonas. Brazil. Hydrol Sci J. 2013;58:1495-504

18. Wampfler R, Mwingira F, Javati S, Robinson L, Betuela I, Siba P, et al. Strategies for detection of Plasmodium species gametocytes. PLoS One. 2013;8:e76316

19. Ministério da Saúde. Guia prático de tratamento da malária no Brasil. Brasília: Secretaria de Vigilância em Saúde; 2010. http://bvsms.saude.gov.br/ bvs/publicacoes/guia_pratico_malaria.pdf. Accessed 15 June 2015. 
20. World Health Organization. Basic Malaria Microscopy Pt.1 Learner's guide. Geneva: WHO Press; 2010.

21. Ministério da Saúde. Manual de Diagnóstico Laboratorial da Malária. Brasilia: Secretaria de Vigilância em Saúde; 2009. http://bvsms.saude.gov.br/bvs/ publicacoes/manual_diagnostico_laboratorial_malaria_2ed.pdf. Accessed 15 June 2015.

22. Rosanas-Urgell A, Mueller D, Betuela I, Barnadas C, Iga J, Zimmerman PA, et al. Comparison of diagnostic methods for the detection and quantification of the four sympatric Plasmodium species in field samples from Papua New Guinea. Malar J. 2010;9:361.

23. Perandin F, Manca N, Calderaro A, Piccolo G, Galati L, Ricci L, et al. Development of a real-time PCR assay for detection of Plasmodium falciparum, Plasmodium vivax, and Plasmodium ovale for routine clinical diagnosis. J Clin Microbiol. 2004;42:1214-9.

24. Ministério da Saúde. Situação epidemiológica da malária no Brasil, 2000 a 2011. Brasil: Secretaria de Vigilância em Saúde; 2013. http://bvsms.saude.gov. br/bvs/periodicos/boletim_epidemiologico_numero_1_2013.pdf. Accessed 3 Aug 2015.

25. Suárez-Mutis MC, Coura JR. Changes in the epidemiological pattern of malaria in a rural area of the middle Rio Negro, Brazilian Amazon: a retrospective analysis. Cad Saúde Pública. 2007;23:795-804.

26. Tada MS, Marques RP, Mesquita E, Martha RCD, Rodrigues JA, Costa JDAN, et al. Urban malaria in the Brazilian Western Amazon Region I. High prevalence of asymptomatic carriers in an urban riverside district is associated with a high level of clinical malaria. Mem Inst Oswaldo Cruz. 2007;102:263-9.

27. Putaporntip C, Hongsrimuang T, Seethamchai S, Kobasa T, Limkittikul K, Cui $L$, et al. Differential prevalence of Plasmodium infections and cryptic Plasmodium knowlesi malaria in humans in Thailand. J Infect Dis. 2009;199: $1143-50$.

28. Waltmann A, Darcy AW, Harris I, Koepfli C, Lodo J, Vahi V, et al. High rates of asymptomatic, sub-microscopic Plasmodium vivax infection and disappearing Plasmodium falciparum malaria in an area of low transmission in Solomon Islands. PLoS Negl Trop Dis. 2015;9:e0003758

29. Tadei WP, Thatcher BD. Malaria vectors in the Brazilian Amazon: Anopheles of the subgenus Nyssorhynchus. Rev Inst Med Trop. 2000;42:87-94.

30. Robinson LJ, Wampfler R, Betuela I, Karl S, White MT, Li Wai Suen CS, et al. Strategies for understanding and reducing the Plasmodium vivax and Plasmodium ovale hypnozoite reservoir in Papua New Guinean children: a randomised placebo-controlled trial and mathematical model. PLoS Med. 2015;12:e1001891.

31. Douglas NM, Ja S, Phyo AP, Siswantoro H, Hasugian AR, Kenangalem E, et al. Gametocyte dynamics and the role of drugs in reducing the transmission potential of Plasmodium vivax. J Infect Dis. 2013;208:801-12.

32. Koepfli C, Robinson L, Rarau P, Salib M, Sambale N, Wampfler R, et al. Blood-stage parasitaemia and age determine Plasmodium falciparum and P. vivax gametocytaemia in Papua New Guinea. PloS One. 2015;10:e0126747.

33. Bousema T, Drakeley C. Epidemiology and infectivity of Plasmodium falciparum and Plasmodium vivax gametocytes in relation to malaria control and elimination. Clin Microbiol Rev. 2011;24:377-410.

34. Kuamsab N, Putaporntip C, Pattanawong U, Jongwutiwes S. Simultaneous detection of Plasmodium vivax and Plasmodium falciparum gametocytes in clinical isolates by multiplex-nested RT-PCR. Malar J. 2012;11:190.

35. Lima NF, Bastos MS, Ferreira MU. Plasmodium vivax: reverse transcriptase real-time PCR for gametocyte detection and quantitation in clinical samples. Exp Parasitol. 2012;132:348-54.

36. Rosas-Aguirre A, Llanos-Cuentas A, Speybroeck N, Cook J, Contreras-Mancilla J, Soto V, et al. Assessing malaria transmission in a low endemicity area of north-western Peru. Malar J. 2013;12:339.

37. Alves FP, Gil LHS, Marrelli MT, Ribolla PEM, Camargo EP, Hildebrando L, et al. Asymptomatic carriers of Plasmodium spp . as infection source for malaria vector mosquitoes in the Brazilian Amazon. J Med Entomol. 2005;42:777-9.

38. Cheng $Q$, Cunningham J, Gatton ML. Systematic review of sub-microscopic P. vivax infections: prevalence and determining factors. PLoS Negl Trop Dis. 2015:9:e3413.

39. Moreira CM, Abo-Shehada M, Price RN, Drakeley CJ. A systematic review of sub-microscopic Plasmodium vivax infection. Malar J. 2015;14:360.

\section{Submit your next manuscript to BioMed Central and we will help you at every step:}

- We accept pre-submission inquiries

- Our selector tool helps you to find the most relevant journal

- We provide round the clock customer support

- Convenient online submission

- Thorough peer review

- Inclusion in PubMed and all major indexing services

- Maximum visibility for your research

Submit your manuscript at www.biomedcentral.com/submit 